\title{
Phase behaviour of hard board-like particles
}

DOI:

10.1039/C7SM00726D

\section{Document Version}

Accepted author manuscript

Link to publication record in Manchester Research Explorer

\section{Citation for published version (APA):}

Cuetos, A., Dennison, M., Masters, A., \& Patti, A. (2017). Phase behaviour of hard board-like particles. Soft Matter. https://doi.org/10.1039/C7SM00726D

\section{Published in:}

Soft Matter

\section{Citing this paper}

Please note that where the full-text provided on Manchester Research Explorer is the Author Accepted Manuscript or Proof version this may differ from the final Published version. If citing, it is advised that you check and use the publisher's definitive version.

\section{General rights}

Copyright and moral rights for the publications made accessible in the Research Explorer are retained by the authors and/or other copyright owners and it is a condition of accessing publications that users recognise and abide by the legal requirements associated with these rights.

\section{Takedown policy}

If you believe that this document breaches copyright please refer to the University of Manchester's Takedown Procedures [http://man.ac.uk/04Y6Bo] or contact uml.scholarlycommunications@manchester.ac.uk providing relevant details, so we can investigate your claim.

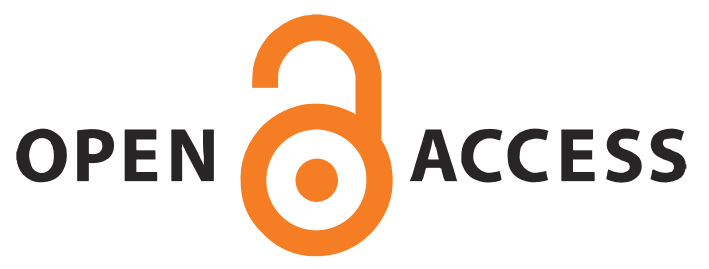




\section{Accepted Manuscript}

This article can be cited before page numbers have been issued, to do this please use: A. Cuetos, M. Dennison, A. J. Masters and A. Patti, Soft Matter, 2017, DOI: 10.1039/C7SM00726D.

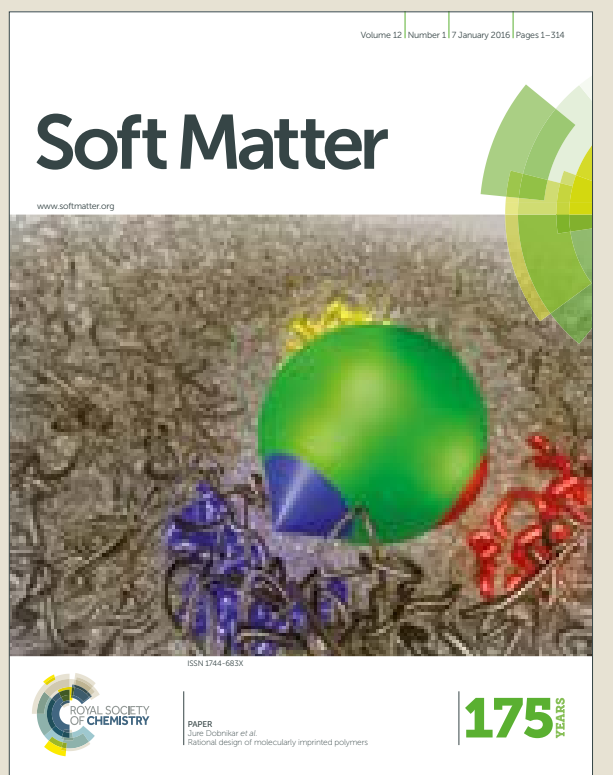

This is an Accepted Manuscript, which has been through the Royal Society of Chemistry peer review process and has been accepted for publication.

Accepted Manuscripts are published online shortly after acceptance, before technical editing, formatting and proof reading. Using this free service, authors can make their results available to the community, in citable form, before we publish the edited article. We will replace this Accepted Manuscript with the edited and formatted Advance Article as soon as it is available.

You can find more information about Accepted Manuscripts in the author guidelines.

Please note that technical editing may introduce minor changes to the text and/or graphics, which may alter content. The journal's standard Terms \& Conditions and the ethical guidelines, outlined in our author and reviewer resource centre, still apply. In no event shall the Royal Society of Chemistry be held responsible for any errors or omissions in this Accepted Manuscript or any consequences arising from the use of any information it contains. 


\title{
Journal Name
}

\section{ARTICLE TYPE}

Cite this: DOl: 10.1039/xxxxxxxxxx

\begin{abstract}
Received Date
Accepted Date
\end{abstract}

DOI: 10.1039/xxxxxxxxxx

www.rsc.org/journalname

\section{Phase behaviour of hard board-like particles ${ }^{\dagger}$}

\author{
Alejandro Cuetos, ${ }^{a}$ Matthew Dennison, ${ }^{b}$ Andrew Masters, ${ }^{c}$ and Alessandro Patti ${ }^{\star c}$
}

We examine the phase behaviour of colloidal suspensions of hard board-like particles (HBPs) as a function of their shape anisotropy, and observe a fascinating spectrum of nematic, smectic and columnar liquid-crystalline phases, whose formation is entirely driven by excluded volum : effects. We map out the phase diagram of short and long HBPs by gradually modifying their shape from prolate to oblate and investigate the long-range order of the resulting morphologies alon, the phase directors and perpendicularly to them. The intrinsic biaxial nature of these particler promotes the formation of translationally ordered biaxial phases, but does not show solid evidenen that it would, per se, promote the formation of the biaxial nematic phase. Our simulations sh $\mathrm{c}$ light on the controversial existence of the discotic smectic phase, whose layers are as thick $x$ the minor particle dimension, which is stable in a relatively large portion of our phase diagrams. Additionally, we modify the Onsager theory to describe the isotropic-nematic phase transition $0_{i}$ freely rotating biaxial particles as a function of the particle width, and find a relatively strong firs ${ }^{\dagger}$ order signature, in excellent agreement with our simulations. In an attempt to shed light on the elusive formation of the biaxial nematic phase, we apply this theory to predict the uniaxial-biaxia nematic phase transition and confirm, again in agreement with simulations, the prevailing stability of the translationally ordered smectic phase over the orientationally ordered biaxial nematic phase.

\section{Introduction}

Colloids are two-phase systems consisting of a dispersed phase, usually droplets or particles, suspended in a continuous medium. They are ubiquitous in our everyday life and play a paramount role in a rich variety of technological applications, especially related to food, paint and cosmetic formulations. According to the IUPAC, at least one of the dimensions of the molecules or particles in the dispersed phase should be roughly between $1 \mathrm{~nm}$ and $1 \mu \mathrm{m}^{1}$. Particles of this size experience stochastic collisions with the molecules of the dispersing medium. The resulting random drifting stems from a thermal energy contribution of the order of few $k_{B} T$ per particle and is referred to as Brownian motion, af-

\footnotetext{
${ }^{a}$ Department of Physical, Chemical and Natural Systems, Pablo de Olavide University, 41013 Sevilla, Spain

${ }^{b}$ Institut für Theoretische Physik, Technische Universität Berlin, Hardenbergstrasse 36, 10623 Berlin, Germany.

${ }^{c}$ School of Chemical Engineering and Analytical Science, The University of Manchester, Manchester, M13 9PL, UK; E-mail:alessandro.patti@manchester.ac.uk

$\dagger$ Electronic Supplementary Information (ESI) available. See DOI: 10.1039/b000000x/
}

ter the British botanist Robert Brown. In 1827, Brown observec pollen grains suspended in water and performing persistent anci random jump-like moves ${ }^{2}$. Colloids can be classified accordin to the physical state of both the continuous and dispersed phases For example, emulsions and foams are dispersions of, respective. small liquid and gas droplets in an other liquid. When the dispersed phase consists of very small solid particles suspended in. liquid, the colloid is generally referred to as suspension. A crucial requirement to effectively use colloids in many relevant applications is their thermodynamic and kinetic stability, which is $\mathrm{s}$ large extent determined by the delicate balance between entropic and enthalpic contributions to their mutual interactions. Controlling these interactions by, for example, adding an electrolyte or a polymer that modifies the particle surface or shape, determines a rich phase behaviour and is key to exploiting, e.g., optical, mechanical, and thermal properties of a colloid. As a consequence, colloids can show a very intriguing phase and aggregation behaviour, surprisingly similar to that of molecular and atomic systems. This similarity has a striking relevance in materials science: it is crucial to unveiling a number of dynamical processes, such as 
crystallisation, involving atoms or molecules that are too fast to be detected via conventional microscopy, and provides the opportunity to design functional materials for advanced electro-optical devices ${ }^{3}$.

Colloidal suspensions of anisotropic particles are particularly attractive for a wide spectrum of applications, including, for instance, photonic band gap materials to manipulate the propagation of light and control the emission of photons at visible wavelengths ${ }^{4}$. Although methods to synthesise anisotropic colloidal particles have been known since the 1990s, most of them lacked the features (high yield and shape selectivity) to efficiently exploit technological applications ${ }^{5-7}$. The recognition of the impact of the particle shape anisotropy on engineering materials with novel or improved properties sparked off the development of chemical $^{8}$, physical ${ }^{9}$, and biosynthetic ${ }^{10}$ techniques to fabricate ad hoc building blocks, such as superballs ${ }^{11}$, lock-and-key particles $^{12}$, fused spheres ${ }^{13}$, and cuboids ${ }^{14-17}$. Such novel shapes enabled the further enrichment of the already complex phase behaviour of colloids, which includes liquid crystals (LCs) ${ }^{18}$, equilibrium gels ${ }^{19}$, and porous liquids ${ }^{20}$. Side by side with experiments, theory and simulation have greatly contributed to understanding the effects of shape on the phase behaviour of colloidal suspensions, often offering preliminary screenings through the huge number of experimental variables ${ }^{21-24}$. Very recently, Avendaño and coworkers reported the fascinating phase behaviour of a less conventional family of anisotropic particles: interlocking planar nanorings ${ }^{25}$. These nonconvex colloidal particles are able to form smectic LCs of remarkably high porosity, which have a very promising potential in gas adsorption and storage.

Biaxial colloidal particles, such as bent-core and board-like particles, displaying three orthogonal internal axes, have the potential to form a particularly interesting LC phase, the biaxial nematic phase, $\mathrm{N}_{\mathrm{B}}$, which exhibits an orientational order along three directors and thus two distinct optical axes. Nevertheless, stable $\mathrm{N}_{\mathrm{B}}$ phases are rarely observed because competing structures, such as the smectic (Sm) phase, limit or preclude its existence. Theory indicates that some particle geometries might be more successful than others in observing this phase. In particular, the self-dual shape, a geometry almost exactly in between prolate and oblate, was shown to promote the formation of the $\mathrm{N}_{B}$ phase, although into a relatively limited region of stability ${ }^{26}$. Variational cluster expansion theory and Monte Carlo (MC) simulations applied to binary mixtures of hard lath-shaped particles, perfectly aligned along their major axis, suggested that the stability of the $\mathrm{N}_{\mathrm{B}}$ phase could be enhanced by size dispersity ${ }^{27}$. These preliminary intuitions were later experimentally confirmed by Vroege and coworkers, who observed a remarkably stable $\mathrm{N}_{B}$ phase in colloidal suspensions of board-like goethite particles with selfdual shape ${ }^{28}$. Surprisingly, the range of stability found experimentally significantly exceeded that predicted by previous theo- ries and simulation studies ${ }^{26,27}$ and was later ascribed by Belli et al to the remarkable size dispersity of the goethite particles ${ }^{29}$. The mean-field theory applied by Belli and based on the Zwanzig restricted orientation model ${ }^{30}$, pointed out the crucial role of polydispersity in stabilising the $\mathrm{N}_{\mathrm{B}}$ phase, which was found to be metastable with respect to the Sm phase for monodisperse HBPs. Adding a non-adsorbing depletant to a pure system of HBPs was shown to have a similar effect and enhance the stability of the $\mathrm{N}_{\mathrm{B}}$ phase significantly ${ }^{31}$.

If we restrict our attention to monodisperse systems, many hard biaxial particles investigated over the last twenty years were shown to form stable $\mathrm{N}_{\mathrm{B}}$ phases. For instance, Camp and Allen detected a stable $\mathrm{N}_{\mathrm{B}}$ phase in suspensions of hard biaxial ellipsoids ${ }^{32}$, while Peroukidis and Vanakaras in fluids of hard spheroplatelets ${ }^{33}$. This particular geometry, consisting of board-like particles with rounded corners, was also examined, in the context of the scaled-particle theory (SPT) within the Zwanzig model, by Taylor and Herzfeld ${ }^{26}$. By incorporating translational ordering into the framework of SPT, these authors noticed a remarkable reduction in the stability region of the $\mathrm{N}_{\mathrm{B}}$ phase, which was almost completely replaced by the uniaxial and biaxial $\mathrm{Sm}$ phase. As far as HBPs are concerned, Vanakaras et al observed the existence of the $\mathrm{N}_{B}$ phase in monodisperse systems of perfectly aligned HBPs, which were not allowed to rotate about their two shorter axes ${ }^{27}$. Escobedo and coworkers calculated the phase diagram of freely rotating oblate and prolate parallelepipeds with square cross section and unveiled a rich variety of LC phases, such as the cubaticlike parquet phase, but did not observe the $\mathrm{N}_{B}$ phase ${ }^{34,35}$. The more recent work by Belli et al, using the Zwanzig model, revealed the existence of the $\mathrm{N}_{B}$ phase of HBPs with self-dual shape and length-to-diameter ratio $L / W=3^{31}$. All these computational and theoretical studies suggest that a biaxial geometry is most likely a necessary, but not always sufficient, condition to stabilise biaxial phases. Additional degrees of freedom, such as particle alignment, size and shape dispersity, can dramatically alter the phase behaviour of HBPs. Despite the widespread interest within the LC community, the phase behaviour of monodisperse freelyrotating HBPs is far from being fully understood and the question whether these particles can actually form stable $N_{B}$ phases remains open.

Additionally, our results confirm the existence of the longdebated discotic Sm phase, consisting of layers as thick as the particle minor axis. While the most common Sm phases are characterised by layers as thick as the particle length, we observe the formation of Sm phases whose layers' thickness is given by the minor particle dimension, $T$. This intriguing phase, referred to as discotic or oblate smectic $\left(\mathrm{Sm}^{-}\right) \mathrm{LC}$, was first predicted by Taylor and Herzfeld, who applied scaled particle theory in conjunction with a cell description of translational order to study the phase behaviour of mutually orthogonal hard spheroplatelets ${ }^{26}$. As far 
as hard cuboids are concerned, the $\mathrm{Sm}^{-}$phase was detected in systems of hard parallelepipeds $(L=W=5 T)$ by lattice MC simulations within the restricted-orientation Zwanzig model ${ }^{36}$ and later predicted by the Fundamental Measure Theory (FMT) also in the context of the Zwanzig approximation ${ }^{37,38}$. John and Escobedo performed off-lattice MC simulations of hard tetragonal parallelepipeds with unrestricted orientations and provided a beautiful picture of their rich phase behaviour ${ }^{34,35}$. However, these authors could not confirm the theoretical predictions on the existence of the $\mathrm{Sm}^{-}$phase, suggesting that the stability of this phase could be due to the reduced orientational freedom embedded in the FMT. Our simulation and theoretical findings shed light on the controversial existence of the $\mathrm{Sm}^{-}$, which, at least for the geometries examined here, exhibits a relevant stability and further enriches the already complex phase behaviour of HBPs.

In the present study, we perform extensive MC simulations of freely rotating HBPs to unveil their intriguing phase behaviour, which is characterised by a plethora of liquid crystal phases and peculiar oblate-to-prolate-to-oblate phase transitions. We also propose an expansion of the Onsager theory ${ }^{18}$ to investigate the isotropic-to-nematic and uniaxial-to-biaxial transitions in suspensions of HBPs. While the latter transition is analysed in the context of HBPs perfectly aligned along their major axis, as previously assumed also by other researchers ${ }^{27}$, the isotropic-to-nematic transition does not involve any possible restriction on the orientation of the particles, which are left free to rotate. This is a paramount difference with respect to other theoretical treatments that predicted the isotropic-to-nematic transition by employing the restricted-orientation Zwanzig model, which allows for only six orthogonal orientations ${ }^{29,36-38}$. Unfreezing the particle's degree of freedom is crucial to fully understand the nature of the isotropic-nematic coexistence region, which is found to be significantly larger than that predicted by previous studies on identical ${ }^{29,31}$ or similar ${ }^{26,39}$ geometries, and reveals a significant first-order signature. Moreover, one novel feature of the present theory, as compared to previous virial expansions on axially symmetric particles ${ }^{40}$, is that in the nematic phases, we do not assume free rotation about the aligned molecular axis - i.e. we take into account the molecular biaxiality order parameter.

\section{Simulation}

\subsection{Model}

We perform simulations of systems containing between $N=1100$ and $N=3500$ freely rotating HBPs with width $W$, thickness $T$, and length $L$. In particular, $T$ is kept constant and is the unit length of the systems studied. Two different particle lengths are considered here: $L_{1}^{*} \equiv L_{1} / T=9$ and $L_{2}^{*} \equiv L_{2} / T=12$. Finally, the particle width, $W^{*} \equiv W / T$, varies in the range $1 \leq W_{1}^{*} \leq 9$ and $1 \leq$ $W_{2}^{*} \leq 12$, for particles with reduced length $L_{1}^{*}$ and $L_{2}^{*}$, respectively. By tuning $W^{*}$ in this range, the particle shape gradually changes from a prolate (rod-like) to an oblate (plate-like) geometry, as shown in Fig. 1.

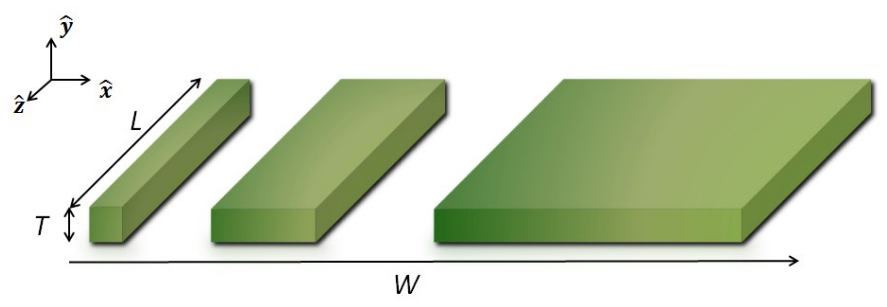

Fig. 1 Model HBPs with thickness $T$, length $L$, and width $W=T, 3 T$, and $L$, from left to right. $T$, the unit length, is kept constant in all the systems studied in this paper. The particle length in this figure is $L=9 T$ but systems containing longer particles $(L=12 T)$ have also been investigated. The particle width is a simulation parameter assuming a spectrum of values between $W=T$ (rod-like, prolate particles) and $W=L$ (plate-like, oblate particles). $\hat{\mathbf{x}}, \hat{\mathbf{y}}$, and $\hat{\mathbf{z}}$ are the unit vectors along $W, T$, and $L$, respectively.

\subsection{Methodology}

We performed MC simulations in the isobaric-isothermal ensem ble constraining a costant number of particles into a rectangula box with periodic boundaries. Phase transitions were usually de termined by gradually expanding a solid crystalline $(\mathrm{K})$ phase up to an isotropic (I) phase, but some systems were also compressec to enhance the accuracy of the phase boundaries. The range of pressures explored is very broad and depends on the particle geometry (see ESI $\dagger$ ). Each MC cycle consisted of $N$ attempts of div placing and/or rotating the randomly selected particles, plus ar attempt to modify the three box lengths independently. Trans lational and rotational moves as well as volume changes wer accepted if no overlap was detected. To test for particle overlaps. we applied the separating axes method described by Gottschalk et al. ${ }^{41}$ and adapted by John and Escobedo to study the phase bo haviour of tetragonal parallelepiped particles with square cross section $^{34}$. We refer the interested reader to the Appendix Ref. $^{34}$ for further details. During the equilibration run, we calcu lated the uniaxial $\left(S_{2}\right)$ and biaxial $\left(B_{2}\right)$ order parameters as $\mathrm{w}_{1}{ }^{\prime}$ as the packing fraction $\eta=N v_{0} / V$, with $v_{0}=T \times W \times L$ and $V$ the volume of the simulation box. The systems were considered to be equilibrated when $\eta, S_{2}$ and $B_{2}$ reached a steady value within reasonable statistical fluctuations.

We have calculated the nematic order parameter and nematic director associated to each molecular axis by applying the standard procedure of diagonalizing a traceless symmetric secondrank tensor defined as

$$
\mathbf{Q}^{\lambda \lambda}=\frac{1}{2 N}\left\langle\sum_{i=1}^{N}\left(3 \hat{\boldsymbol{\lambda}}_{i} \cdot \hat{\boldsymbol{\lambda}}_{i}-\mathbf{I}\right)\right\rangle
$$


where $\hat{\boldsymbol{\lambda}}=\hat{\mathbf{x}}, \hat{\mathbf{y}}, \hat{\mathbf{z}}$ is the unit orientation vector of particle $i$, I the second-rank unit tensor, and the angular brackets indicate ensemble average. Diagonalization of $\mathbf{Q}^{\lambda \lambda}$ provides three eigenvalues and three associated eigenvectors. The nematic order parameter $S_{2}$ is the largest positive eigenvalue of $\mathbf{Q}^{\lambda \lambda}$ and the corresponding eigenvector determines the uniaxial nematic director ${ }^{42}$. In particular, the nematic order parameter $S_{2, L}$ is the largest eigenvalue of the tensor $\mathbf{Q}^{z z}$, while the corresponding eigenvector $\hat{\mathbf{n}}$ is the nematic director associated to the preferential orientation of the particle axis $\hat{\mathbf{z}}$. Similarly, diagonalizing $\mathbf{Q}^{x x}$ and $\mathbf{Q}^{y y}$, the nematic order parameters $S_{2, W}$ and $S_{2, T}$ and the nematic directors $\hat{\mathbf{m}}$ and î can be determined. By employing these same symmetric tensors, three biaxial order parameters can also be calculated ${ }^{32}$. For instance, the biaxial order parameter associated to the nematic director along $L, \hat{\mathbf{n}}$, reads

$$
B_{2, L}=\frac{1}{3}\left(\hat{\mathbf{m}} \cdot \mathbf{Q}^{x x} \cdot \hat{\mathbf{m}}+\hat{\mathbf{l}} \cdot \mathbf{Q}^{y y} \cdot \hat{\mathbf{l}}-\hat{\mathbf{m}} \cdot \mathbf{Q}^{y y} \cdot \hat{\mathbf{m}}-\hat{\mathbf{l}} \cdot \mathbf{Q}^{x x} \cdot \hat{\mathbf{l}}\right)
$$

$B_{2, W}$ and $B_{2, T}$ can be calculated by similar expressions. Following previous works ${ }^{43-45}$, it is sufficient to monitor a single biaxial order parameter to assess phase biaxiality. In other words, we only need to examine the fluctuations of the two particle unit vectors perpendicular to the main nematic director. If, for instance, the particles are on average aligned along their $\hat{\mathbf{z}}$ axis and thus the main nematic director is $\hat{\mathbf{n}}$, then the fluctuations of the other two particle axes, $\hat{\mathbf{x}}$ and $\hat{\mathbf{y}}$, in the plane defined by $\hat{\mathbf{m}}$ and $\hat{\mathbf{l}}$, should be considered. In this case, $S_{2, L}=1$ and $B_{2, L}=0$ would give a perfect uniaxial phase, while $S_{2, L}=1$ and $B_{2, L}=1$ a perfectly aligned biaxial phase. Similar considerations hold if the main nematic director is $\hat{\mathbf{m}}$ or $\mathbf{l}$. In what follows, we refer to the biaxial order parameter associated to the main nematic director as $B_{2}$.

Order parameters, alone, are not sufficient to distinguish between two uniaxial or biaxial phases, such as a nematic $(\mathrm{N})$ from a smectic (Sm) phase. To this end, after equilibration, we addressed the existence and nature of long-range structural correlations by calculating the pair distribution functions along the nematic directors, $g_{\|, L}\left(r_{\|, L}^{*}\right), g_{\|, W}\left(r_{\|, W}^{*}\right)$ and $g_{\|, T}\left(r_{\|, T}^{*}\right)$, and perpendicularly to them, $g_{\perp, L}\left(r_{\perp, L}^{*}\right), g_{\perp, W}\left(r_{\perp, W}^{*}\right)$ and $g_{\perp, T}\left(r_{\perp, T}^{*}\right)$, with $r_{\|}^{*}=r_{\|} / T$ and $r_{\perp}^{*}=r_{\perp} / T$ the longitudinal and transverse distances, respectively, between pairs of particles. Finally, to investigate the local structure of columnar (Col) and $\mathrm{K}$ phases, we have also calculated an order parameter that can distinguish between the relative positions between the particles in planes perpendicular to the nematic director:

$$
\psi_{n}=\frac{1}{N_{j}} \sum_{k}^{N_{j}} \exp \left(\mathrm{i} n \theta_{j k}\right)
$$

where $N_{j}$ is the number of nearest neighbours around particle $k$, $\theta_{j k}$ is the angle formed by the vectors connecting $j$ and $k$ and a reference axis, and $n=4$ or 6 to determine the occurrence of, respectively, quadratic or hexatic symmetry.

\section{Theory}

\subsection{Isotropic-nematic phase transition}

\subsubsection{Uniaxial particles}

The Onsager theory of nematic LCs ${ }^{18}$ is based on an expression for the Helmholtz energy $A$ which contains two competing terms. One arises from the mixing of particles of different orientations, similar to an entropy of mixing, while the other arises from excluded volume interactions and is expressed via a virial expansion. Onsager showed that the Helmholtz energy of a system containing $N$ particles at volume $V$ (and hence with a number density $\rho=N / V$ ), can be given as

$$
a=\frac{\beta A}{N}=\ln \Lambda^{3} \rho-1+\sigma[f(\boldsymbol{\Omega})]+\sum_{n=2}^{\infty} \frac{1}{n-1} B_{n} \rho^{n-1} .
$$

Here, $\beta$ is the inverse temperature, $\ln \Lambda^{3} \rho-1$ is the ideal gas contribution with $\Lambda$ the de Broglie wavelength, and $\sigma[f(\boldsymbol{\Omega})]$ is the entropy of mixing-like term with $f(\boldsymbol{\Omega})$ giving the one-particle orientational distribution function and $\boldsymbol{\Omega}$ the particle orientation. The final term is the virial expansion which corrects for manybody interactions, with $B_{n}$ denoting the $n^{\text {th }}$ virial coefficient. The key component in this expression is the one-particle orientational distribution $f(\boldsymbol{\Omega})$. For uniaxial particles, Onsager used a trial function of the form

$$
f(\boldsymbol{\Omega})=\frac{\alpha \cosh (\alpha \cos \theta)}{4 \pi \sinh \alpha},
$$

where $\theta$ is the angle between the nematic director and the director through the particle symmetry axis and $\alpha$ is a free parameter which describes the degree of nematic ordering, with $\alpha=0$ for isotropic orientational ordering and $\alpha=\infty$ for perfect nematic alignment. $\alpha$ can be related to the nematic order parameter as

$$
\begin{aligned}
S & =\int f(\boldsymbol{\Omega}) P_{2}(\cos \theta) d \boldsymbol{\Omega}, \\
& =1+\frac{3}{\alpha^{2}}-\frac{3}{\alpha \tanh \alpha},
\end{aligned}
$$

where $P_{2}(\cos \theta)=\left(3 \cos ^{2} \theta-1\right) / 2$ is the second Legendre polynomial. This trial function can be used to calculate the entropy of mixing-like term as

$$
\begin{aligned}
\sigma[f(\boldsymbol{\Omega})] & =\int f(\boldsymbol{\Omega}) \ln [4 \pi f(\boldsymbol{\Omega})] d \boldsymbol{\Omega}, \\
& =\ln \left(\frac{\alpha}{\tanh (\alpha)}\right)+\frac{\tan ^{-1}(\sinh \alpha)}{\sinh \alpha}-1,
\end{aligned}
$$


where $d \boldsymbol{\Omega}=\sin \theta d \theta d \phi$, with $\phi$ the azimuthal angle. The virial coefficients can be calculated from

$$
B_{n}=\frac{1-n}{n ! V} \int \ldots \int f\left(\boldsymbol{\Omega}_{1}\right) \ldots f\left(\boldsymbol{\Omega}_{n}\right) V_{n} d \boldsymbol{\Omega}_{1} \ldots d \boldsymbol{\Omega}_{n} d \mathbf{r}_{1} \ldots d \mathbf{r}_{n},
$$

with $V$ the volume, and $\mathbf{r}_{i}$ and $\boldsymbol{\Omega}_{i}$ the position and orientation of particle $i . V_{n}$ is given as

$$
V_{n}=\sum_{S_{n}} \prod_{i<j}^{n} f_{i j} .
$$

The sum over $S_{n}$ denotes the sum over all star integrals with $n$ points (see e.g. Refs. ${ }^{46-48}$ ), and $f_{i j}$ is the Mayer f-bond between particles $i$ and $j$, which for hard particles is -1 when the particles overlap, and 0 when they do not. The virial coefficients can be calculated using MC integration for a range of $\alpha$ values, which gives the Helmholtz free energy entirely as a function of density $\rho$ and $\alpha$. By minimizing Eq. (4) with respect to $\alpha$ for a given density, we obtain the free energy and degree of nematic ordering as a function of $\rho$, from which we can also calculate the pressure as

$$
P=\rho+\sum_{n=2}^{\infty} B_{n} \rho^{n},
$$

and the chemical potential as

$$
\mu=\ln \Lambda^{3} \rho+\sigma[f(\boldsymbol{\Omega})]+\sum_{n=2}^{\infty} \frac{n}{n-1} B_{n} \rho^{n-1} .
$$

Coexistence between the I and $\mathrm{N}$ phases can be located by equating these two properties in the I and $\mathrm{N}$ phases; that is

$$
\begin{aligned}
& P\left(\rho_{I}\right)=P\left(\rho_{N}\right), \\
& \mu\left(\rho_{I}\right)=\mu\left(\rho_{N}\right),
\end{aligned}
$$

where $\rho_{I, N}$ are the isotropic and nematic coexistence densities.

\subsubsection{Biaxial particles}

The above calculations are relatively straighforward for uniaxial particles, and the Onsager trial function given in Eq. (5) allows for Eq. (7) to be solved analytically. Furthermore, when calculating the virial coefficients, it is very computationally efficient to weight the particle orientations generated for the MC integration procedure by $f(\boldsymbol{\Omega})$, and we can obtain an analytical expression from Eq. (5) to pick random numbers from the distribution for a given $\alpha$ value.

For biaxial particles, the particle orientation is no longer described simply by the polar and azimuthal angles, but by the three Euler angles $\theta, \phi$ and $\gamma$, the third of which denotes rotation around the long axis of the particle. A uniaxial $\mathrm{N}$ phase of biaxial particles must take into account ordering of $\theta$ and $\gamma$ (with additional ordering of $\phi$ denoting biaxial nematic ordering). Since the Onsager trial function only accounts for ordering of $\theta$, we therefore require an additional term in the distribution which also takes $\gamma$ into account. We choose the following trial function

$$
f(\boldsymbol{\Omega})=\frac{\cosh (\alpha \cos \theta) \cosh (\beta \sin \theta \cos \gamma)}{C},
$$

where $\alpha$ and $\beta$ are free parameters describing the degree of ordering and $C$ is a normalization parameter defined by $\int f(\boldsymbol{\Omega}) d \boldsymbol{\Omega}=1$. This can then be inserted into Eqs. (7) and (8) to obtain $\sigma$ ard the virial coefficients as a function of $\alpha$ and $\beta$, from which the Helmholtz free energy can be obtained from Eq. (4). By then minimizing $a$ with respect to $\alpha$ and $\beta$ at a fixed density $\rho$, and calculating the pressure and chemical potential from Eqs. (10) and. (11), we can obtain the I-N coexistence densities from Eq. (12) The integrals involving Eq. (13) cannot, to our knowledge, be solved analytically, so instead we must use numerical integratic: and interpolation to calculate $\sigma$ and $B_{n}$ as continuous functions of $\alpha$ and $\beta$.

\subsection{Uniaxial-biaxial nematic phase transition}

In order to describe the transition from a uniaxial to a biaxial nematic phase $\left(\mathrm{N}_{\mathrm{U}}-\mathrm{N}_{\mathrm{B}}\right.$ transition), we must describe the alignmen of the minor axis of the biaxial particle, which is defined by the azimuthal angle $\phi$. A full description of the phase transition wour require a form of the one particle distribution function which de pends on all three of the Euler angles. In practice we find that, for the type of particles considered here, the $\mathrm{N}$ phase is highly $\mathrm{o}^{*}$ dered, and hence we examine $\mathrm{N}_{U}-\mathrm{N}_{\mathrm{B}}$ transitions from a perfectiy aligned nematic phase to a $\mathrm{N}_{B}$ phase. That is, from a translationally disordered phase where the particle long axes are perfectlv: aligned but where the short axes are disordered, to a translationally disordered phase where the particle long axes are perfect:aligned and the short axes show a degree of alignment. Thu we can describe the phase via a one particle distribution functic $r$ which depends only on $\phi$ and a single free parameter $\alpha$, which we choose to be the following

$$
f(\boldsymbol{\Omega})=\frac{\alpha \exp \left(\frac{\alpha|2 \phi-\pi|}{\pi}\right)}{4 \pi\left(e^{\alpha}-1\right)} .
$$

This can be inserted into Eqs. (7) and (8) to obtain $\sigma$ and the virial coefficients as a function of $\alpha$, and we follow the above procedure to obtain the uniaxial and biaxial nematic coexistence densities. 


\subsection{Nematic-smectic and nematic-columnar phase transi- tion}

$\mathrm{N}_{\mathrm{B}}$ phases are often unstable with respect to smectic or columnar phases, and hence we also examine the N-Sm and N-Col phase transitions. We use a bifurcation analysis to calculate the density at which a perfectly aligned nematic phase first becomes unstable with respect to smectic or columnar ordering. We may calculate $\sigma$ for positionally ordered phases by

$$
\sigma=\int \rho(\mathbf{r}) \ln [\rho(\mathbf{r})] d \mathbf{r},
$$

where $\mathbf{r}$ is the particle position. The virial coefficients can be calculated from

$$
B_{n}=\frac{1-n}{n ! V} \int \ldots \int \rho\left(\mathbf{r}_{1}\right) \ldots \rho\left(\mathbf{r}_{n}\right) V_{n} d \mathbf{r}_{1} \ldots d \mathbf{r}_{n},
$$

where $V_{n}$ is given by Eq. (9). Following Ref. ${ }^{49}$, we define the following expression for the density $\rho$ of a smectic phase

$$
\rho=\rho_{0}(1+\varepsilon \xi(\mathbf{r})),
$$

where $\rho_{0}$ is the average density of the system, $\varepsilon$ is a small constant and $\xi(\mathbf{r})$ for the smectic phase is given by

$$
\xi(\mathbf{r})=\cos (k z+\delta),
$$

where $k=2 \pi / d$, with $d$ the spacing between smectic layers, and $\delta$ is a phase shift. For the columnar phase $\xi(\mathbf{r})$ is

$$
\xi(\mathbf{r})=\frac{\cos (k x+\delta)+\cos (k y+\delta)}{2},
$$

where the symbols have the same meaning as before and $d$ is now the spacing between the columns. We make the assumption that the columns form a square $2 \mathrm{~d}$-lattice which has an even spacing of columns in both directions of the columnar plane, which is valid based on our simulation results.

These are then inserted into Eqs. (15) and (16), which are in turn inserted into Eq. (4) to obtain the Helmholtz free energy. This gives us an expansion in powers of $\varepsilon$, with

$$
a=a_{0}+\varepsilon a_{1}+\varepsilon^{2} a_{2} .
$$

Here, the zeroth order term in $\varepsilon, a_{0}$, corresponds to the free energy of the nematic phase and the first order term $a_{1}$ can be shown to be equal to 0 . The second order term $a_{2}$ is given by

$$
a_{2}=\frac{\rho}{2} \int \xi(\mathbf{r})^{2} d \mathbf{r}+\sum_{n=2}^{\infty} \frac{1}{n} B_{n}^{\prime} \rho^{n},
$$

where $B_{n}^{\prime}$ is related to the virial coefficients and is given by

$$
B_{n}^{\prime}=\frac{1-n}{n ! V} \int \ldots \int \zeta V_{n} d \mathbf{r}_{1} \ldots d \mathbf{r}_{n}
$$

where $V_{n}$ is given by Eq. (9) and $\zeta$ is

$$
\zeta=\frac{1}{n(n-1)} \sum_{i=1}^{n-1} \sum_{j=i+1}^{n} \xi\left(\mathbf{r}_{i}\right) \xi\left(\mathbf{r}_{j}\right) .
$$

The term $a_{2}$ will be positive at low densities and negative at high densities. The density at which $a_{2}=0$ corresponds to the point at which the positionally ordered phases will first become stable with respect to the positionally disordered nematic phase. We therefore calulate $a_{2}$ and locate the density at which it is equal to zero, giving us the nematic-smectic instability density $\rho_{S m}$ and the nematic-columnar instability density $\rho_{C o l}$.

\section{Results}

In this section, we analyse and discuss the rich phase behaviour observed in colloidal suspensions of HBPs of length $L_{1}^{*}=9$ and $L_{2}^{*}=12$. In particular, we first present the order parameters and radial distribution functions to accurately distinguish the phases found at equilibrium, and then the phase diagrams and equations of state as obtained by simulation and theory.

\subsection{Phase Characterisation}

The initial configurations for the simulations consisted of particles arranged in a cubic-like lattice with a highly degree of orientational and translational order. These systems were first relaxed at high pressure to determine the stability region of the $\mathrm{K}$ phase and then gradually expanded to liquid-crystalline and isotropic phases. In some cases, such a sequence of isobaric paths was inverted to improve our calculations and thus better locate the phase boundaries. We characterised the phases at equilibrium by calculating the order parameters and radial distribution functions. The evolution of the uniaxial order parameters $S_{2, L}, S_{2, T}$ and $S_{2, W}$, and the biaxial order parameter $B_{2}$, in systems containing HBPs with length $L_{1}^{*}$ and $L_{2}^{*}$ is presented, respectively, in Figs. 2 and 3 as a function of the packing fraction $\eta$. Each frame in both figures refers to a different particle width, decreasing from frame (a) to (d) and producing a transition from oblate (platelike) to prolate (rod-like) anisotropies. In particular, uniaxial and biaxial order parameters are extremely low and basically indistinguishable in the I phase. At larger packing fractions, whose precise value depends on the particle architecture, but generally in the range $\eta=(0.2,0.35)$, one of the uniaxial order parameters $S_{2}$ increases significantly, indicating the occurrence of a $\mathrm{N}$ phase. Oblate $\left(\mathrm{N}^{-}\right)$and prolate $\left(\mathrm{N}^{+}\right)$nematic phases show a surprisingly large uniaxial order parameter along their main nematic director ( $\hat{\mathbf{n}}$ or $\hat{\mathbf{l}}$ ) in the range $S_{2}=(0.6,0.9)$, but usually $S_{2}>0.8$. This sound difference clearly distinguishes the $\mathrm{N}$ phase from the I phase.

At substantially larger packing fractions, the behaviour of both uniaxial and biaxial order parameters becomes strongly dependent on the particle shape anisotropy and therefore on the phases at equilibrium. For instance, in Fig. 2a (oblate HBPs), $B_{2}$ in- 


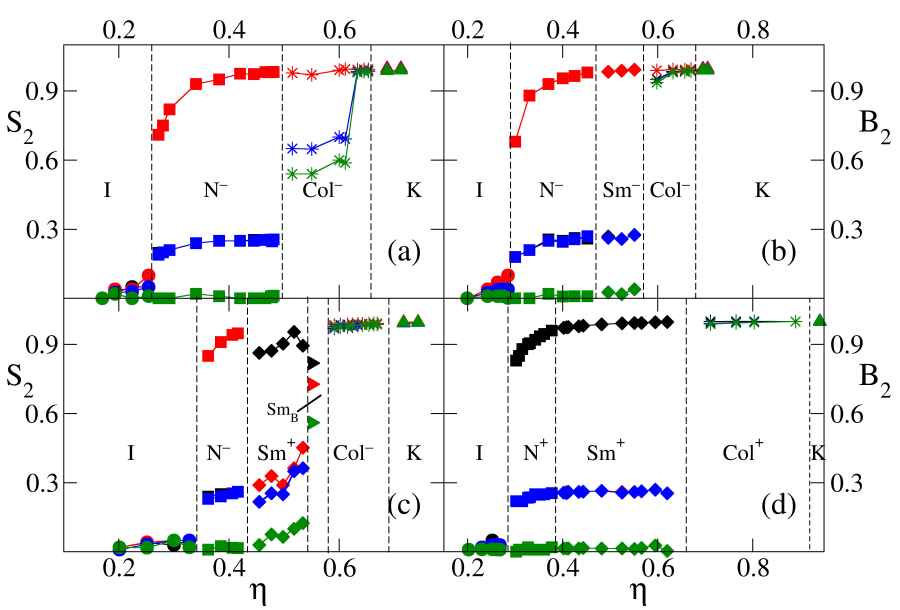

Fig. 2 Uniaxial order parameters as a function of the packing fraction for colloidal suspensions of HBPs with length $L_{1}^{*}$ and width $W_{1}^{*}=9(\mathrm{a}), 7$ (b), 4.5 (c), and 1 (d). Black, red, and blue symbols refer to $S_{2, L}, S_{2, T}$, and $S_{2, W}$, respectively, while the green symbols refer to the dominant biaxial order parameter. The vertical dashed lines indicate phase transitions.

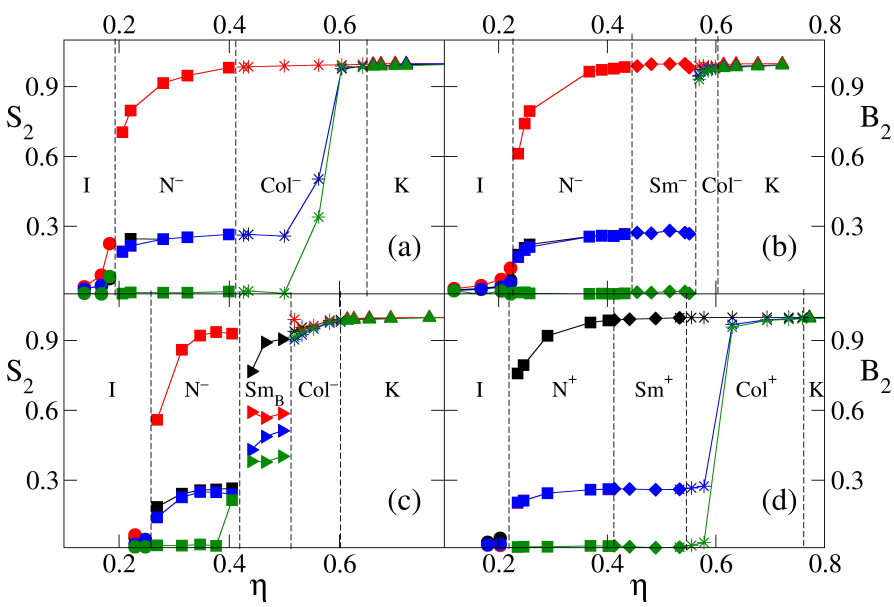

Fig. 3 Uniaxial order parameters as a function of the packing fraction for colloidal suspensions of HBPs with length $L_{2}^{*}$ and width $W_{2}^{*}=12$ (a), 8 (b), 5 (c), and 1 (d). Black, red, and blue symbols refer to $S_{2, L}, S_{2, T}$, and $S_{2, W}$, respectively, while the green symbols refer to the dominant biaxial order parameter. The vertical dashed lines indicate phase transitions.

creases abruptly from 0.01 to approximately 0.54 at $\eta=0.515$, marking the transition from an oblate nematic $\left(\mathrm{N}^{-}\right)$to an oblate columnar $\left(\mathrm{Col}^{-}\right)$phase. By contrast, in Fig. 2d (prolate HBPs), the same biaxial order parameter does not show any noticeable change across the I- $\mathrm{N}^{+}$and $\mathrm{N}^{+}-\mathrm{Sm}^{+}$phase transitions and only increases at $\eta>0.7$, where a prolate columnar $\left(\mathrm{Col}^{+}\right)$phase is formed. Interestingly enough, the largest and smallest uniaxial order parameters can also undergo a mutual inversion as a result of an oblate-prolate transition and consequent rearrangement of the nematic directors (see Fig. 2c). At very large packing frac- tions, all the order parameters assume values above 0.9 and a $\mathrm{K}$ phase is formed.

To unambiguously determine the degree of positional order within a given LC and thus distinguish between N, Sm, Col and $\mathrm{K}$ phases, we calculated the pair distribution functions in the directions parallel and perpendicular to the nematic directors. In particular, the $g_{\|, L}(r)$ in the top frame of Fig. 4 reveals the typical layered structure of a prolate smectic $\left(\mathrm{Sm}^{+}\right)$phase and clearly distinguishes it from a $\mathrm{N}^{+}$phase, whose $g_{\|, L}(r)$, by contrast, is essentially flat (dashed line in the same frame). $\mathrm{In} \mathrm{Sm}^{+}$phases, it is possible to find transverse (interlayer) particles that are trapper in between neighbouring layers (see top-right snapshot in Fig 4), a scenario also detected in colloidal suspensions of uniaxia: prolate particles ${ }^{50-52}$. Nevertheless, the probability of observing such transverse particles is so low that the corresponding radial distribution function cannot actually reveal their presence.

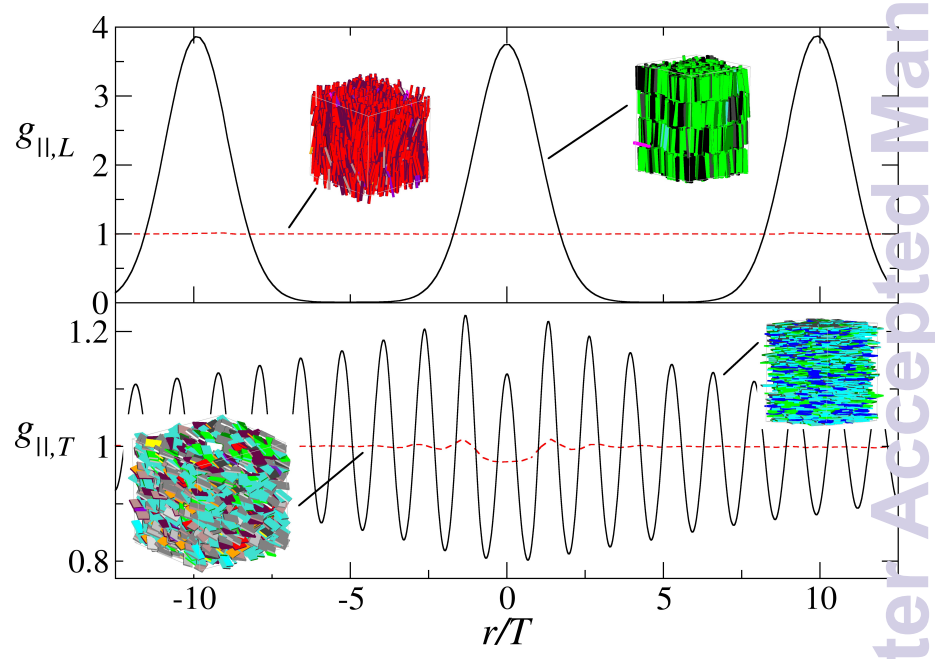

Fig. 4 Parallel pair distribution functions along the main nematic director of prolate (top) and oblate (bottom) smectic liquid crystals of HBPs with $L_{1}^{*}$ and $W_{1}^{*}=1$ (top), and $L_{1}^{*}$ and $W_{1}^{*}=5.5$ (bottom). Dashed and solid lines refer to the $g_{\|}(r)$ calculated in the nematic and smectic phase, respectively. Packing fractions: $0.37\left(\mathrm{~N}^{+}\right), 0.525\left(\mathrm{Sm}^{+}\right), 0.400\left(\mathrm{~N}^{-}\right)$, ar $0.493\left(\mathrm{Sm}^{-}\right)$. The insets show typical equilibrium configurations of eaci phase, with the colour gradient indicating the orientation of the particle major axis.

The radial distribution function displayed in the bottom frame of Fig. 4 (solid line) reveals a different pattern, where periodic peaks are indentified at multiples of the particle thickness. Although the layer thickness of the most recurrent Sm phases is determined by the particle length, here we find a Sm phase whose layers are as thick as the minor particle dimension, $T$, referred to as discotic or oblate smectic phase $\left(\mathrm{Sm}^{-}\right)$. The existence of this phase has been rather controversial over the last decade as evidenced by the lack of full agreement between FMT predictions ${ }^{37,38}$ and MC simulations ${ }^{34,35}$. The results shown here indi- 
cate that the $\mathrm{Sm}^{-}$phase is remarkably stable across a wide range of particle's geometries.

Oblate and prolate Sm phases can also be distinguished from each other by analysing the relevant uniaxial order parameters, which are $S_{2, T}$ for the former and $S_{2, L}$ for the latter. In particular, for $\mathrm{Sm}^{-}$phases $S_{2, T}>S_{2, L}$, whereas for $\mathrm{Sm}^{+}$phases $S_{2, L}>S_{2, T}$, as observed, respectively, in frames (b) and (d) of Fig. 2 or Fig. 3. The existence of $\mathrm{Sm}^{-}$and $\mathrm{Sm}^{+}$phases suggests that, for intermediate geometries between the rod-like and plate-like shape, a biaxial smectic $\left(\mathrm{Sm}_{\mathrm{B}}\right)$ phase, similar to what previously detected in systems of hard perfect tetragonal parallelepipeds ${ }^{35}$, might also exist. This is indeed the case for the two particle lengths, $L_{1}^{*}$ and $L_{2}^{*}$, investigated here. As a matter of fact, the uniaxial order parameters in Fig. 2c and Fig. 3c, which refer, respectively, to the particle geometries $\left(L_{1}^{*}, W_{1}^{*}\right)=(9,4.5)$ and $\left(L_{2}^{*}, W_{2}^{*}\right)=(12,5)$, unveil a strong orientational correlation between each of the three particle axes and confirm the formation of a $\mathrm{Sm}_{\mathrm{B}}$ phase at $0.55<\eta<0.57$ for the shortest and $0.42<\eta<0.45$ for the longest HBPs. The existence of the $\mathrm{Sm}_{\mathrm{B}}$ phase is further confirmed by the analysis of the biaxial order parameters, $B_{2}$, provided in the same figures along with those of $\mathrm{Sm}^{-}$and $\mathrm{Sm}^{+}$ phases for comparison. The $\mathrm{Sm}_{\mathrm{B}}$ is a very peculiar phase with long-ranged translational order in the direction of the particle major axis, as the $\mathrm{Sm}^{+}$phase, and long-ranged orientational order along three directions. Within each layer, there is not significant positional order and the in-layer pair distribution function decays to 1 at relatively short distances (not shown here). This lack of in-layer positional order distinguishes the $\mathrm{Sm}_{\mathrm{B}}$ phase from a crystal phase. Typical configurations of $\mathrm{Sm}^{-}$and $\mathrm{Sm}_{\mathrm{B}}$ phases of HBPs are shown in Fig. 5. A visual inspection of the top view of the $\mathrm{Sm}_{\mathrm{B}}$ phase seems to indicate the occurrence of a quasi-long-range orientational order along two perpendicular axes, resembling the tetratic arrangement of hard-rectangle ${ }^{53}$ and hard-square ${ }^{54}$ systems. Our preliminary calculations would confirm this scenario, but further investigation is needed to provide a more complete insight into this interesting behaviour.

At increasing packing fractions, our HBPs start to self-assemble into $\mathrm{Col}^{-}$or $\mathrm{Col}^{+}$phases. While in the $\mathrm{Col}^{-}$phase the particles are arranged in stacks oriented along their minor axis, the $\mathrm{Col}^{+}$ phase is made of columns incorporating prolate particles oriented along their major axis. The pair distribution functions of representative $\mathrm{Col}^{-}$and $\mathrm{Col}^{+}$phases are shown in Fig. 6. Frame (a) refers to particles of length $L_{1}^{*}$ and width $W_{1}^{*}=8$, and frame (b) to particles of length $L_{1}^{*}$ and width $W_{1}^{*}=2$. Both frames display the $g_{\|}(r)$ calculated in the direction of the three nematic directors $\hat{\mathbf{n}}$ (solid lines), $\hat{\mathbf{m}}$ (dot-dashed lines), and î (dashed lines). The intra-column structural correlations are very weak, typical of a liquid-like system, while the inter-column correlations are relatively strong and indicate a long-range ordered arrangement with the particles roughly located at multiple distances of $W_{1}^{*}=8$ and

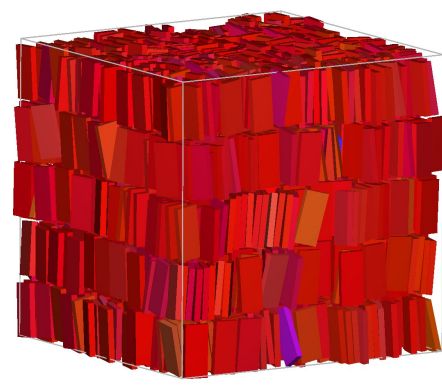

(a)

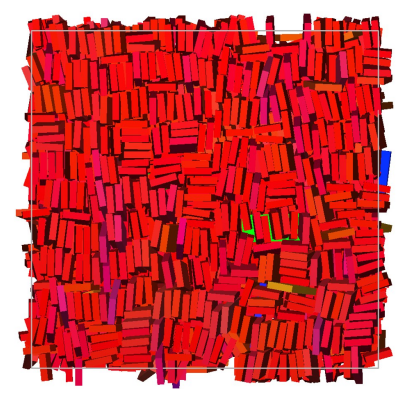

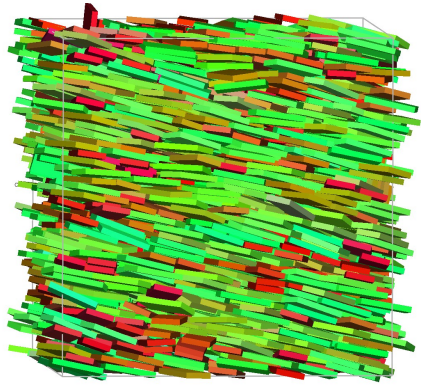

(b)

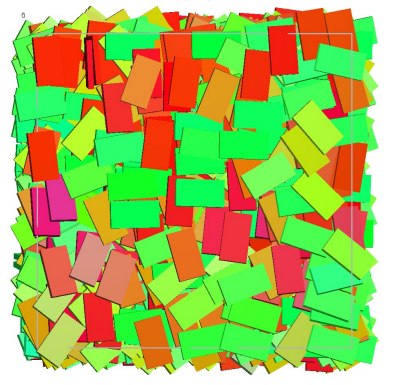

Fig. 5 Front and top views of biaxial (a) and oblate (b) Sm liquid crystals containing $2000 \mathrm{HBPs}$ of length $L_{1}^{*}$ and width $W_{1}^{*}=4$, and $L_{1}^{*}$ and width $W_{1}^{*}=5$, respectively. Packing fractions: 0.570 (a) and 0.467 (b). The colour gradient follows the orientation of the particle major axis.

$L_{1}^{*}=9$ (top frame) or $W_{1}^{*}=2$ and $T_{1}^{*}=1$ (bottom frame) from each other. In the top frame, the slight shift of the peak between $g_{\|, \hat{\mathbf{n}}}(r)$ and $g_{\|, \hat{\mathbf{m}}}(r)$ might indicate a preferential particle orientation in the planes perpendicular to the columns, a sort of alignment between identical faces of the particles. To better address this point, we quantified the angular correlation between the particle unit vectors perpendicular to $\mathbf{i}$ by calculating the orientation distribution function $\chi(\hat{\mathbf{x}}, \hat{\mathbf{z}})=\left\langle\left(3 \hat{\mathbf{x}}_{i} \cdot \hat{\mathbf{z}}_{j}-1\right) / 2\right\rangle$, where $\langle\ldots\rangle$ indicates ensemble average over all measured orientations of particle pairs $(i, j)$. In particular, $\chi(\hat{\mathbf{x}}, \hat{\mathbf{z}}) \simeq 0.1$, indicating a negligible particle alignment across the columns and two equally probable preferential orientations. This picture is not general though. A significant inter-column particle alignment, with a unique preferential orientation, is detected in $\mathrm{Col}^{+}$phases and in $\mathrm{Col}^{-}$phases consisting of HBPs with larger $L / W$ aspect ratios, for instance $\left(L_{1}^{*}, W_{1}^{*}\right)=(9,7.5)$, and, upon crowding, in the $\mathrm{K}$ phase. In these cases, the larger aspect ratio imposes a more efficient packing at similar packing fractions $(\eta \approx 0.6)$ and provides a crystal-like distribution across the columns while maintaining a liquid-like translational order along them. In Fig. 7, we compare the front and top views of two $\mathrm{Col}^{-}$and one $\mathrm{Col}^{+}$phases where the orientation distribution function assumes the values $\chi(\hat{\mathbf{x}}, \hat{\mathbf{z}}) \simeq-0.5$ (Fig. 7a and 7c) and $\chi(\hat{\mathbf{x}}, \hat{\mathbf{z}}) \simeq 0.1$ (Fig. 7b).

Interestingly, the top views of the three snapshots in Fig. 7 unveil an inter-columnar quadratic arrangement that has been con- 


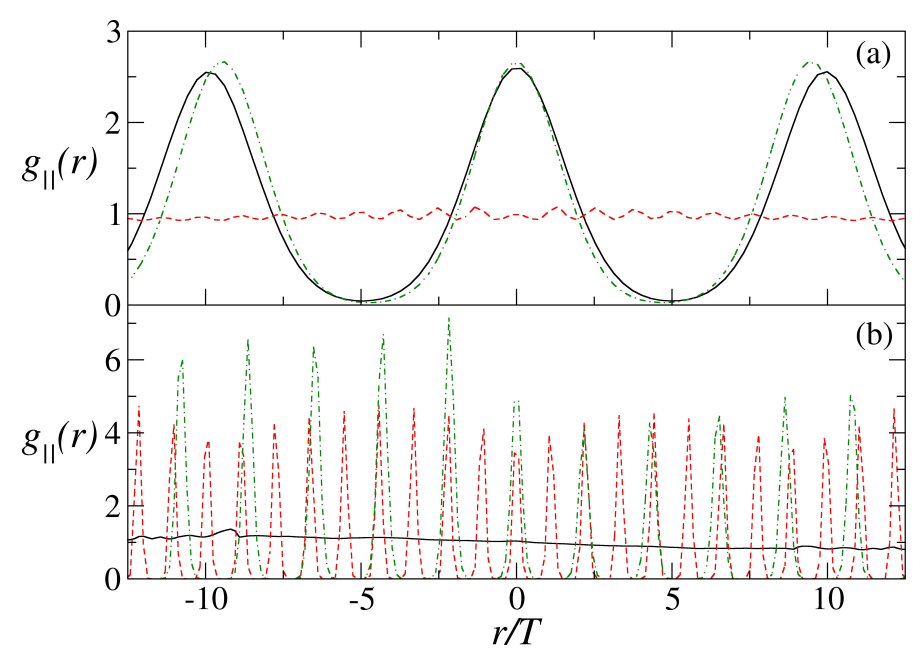

Fig. 6 Frame (a): parallel pair distribution functions of an oblate columnar liquid crystal phase containing $2000 \mathrm{HBPs}$ of length $L_{1}^{*}$ and width $W_{1}^{*}=8$ at $\eta=0.597$. Frame (b): parallel pair distribution functions of a prolate columnar liquid crystal phase containing $2000 \mathrm{HBPs}$ of length $L_{1}^{*}$ and width $W_{1}^{*}=2$ at $\eta=0.809$. Solid, dot-dashed and dashed lines refer to the directions parallel to the nematic directors $\hat{\mathbf{n}}, \hat{\mathbf{m}}$ and $\hat{\mathbf{l}}$, respectively.

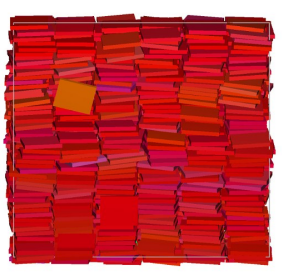

(a)

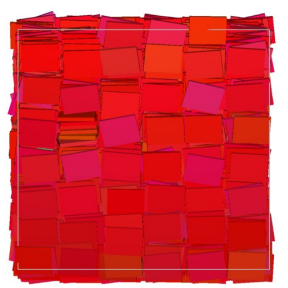

Fig. 7 Front and top views of oblate $(a, b)$ and prolate (c) columnar liquid crystals of HBPs with length $L_{1}^{*}$ and width $W_{1}^{*}=7.5(\mathrm{a}), W_{1}^{*}=8(\mathrm{~b})$, and $W_{1}^{*}=2$ (c). Packing fractions: $\eta=0.590$ (a), $\eta=0.597$ (b), and $\eta=0.809$ (c). The colour gradient follows the orientation of the particle major axis.

sistently observed in all the Col phases found in this study. To characterise this four-fold symmetry and unambiguously distinguish it from an hexagonally-packed arrangement, we calculated the quadratic, $\psi_{4}$, and hexatic, $\psi_{6}$, order parameters. In Fig. 8, these two order parameters are presented as a function of the packing fraction $\eta$ for a number of systems at $L_{1}^{*}$ and $W_{1}^{*}=7.5$ and 8, including the $\mathrm{Col}^{-}$phases shown in Fig. 7a and 7b. While both parameters are almost negligible and indistinguishable in the I phase, at increasing packing fractions it becomes evident that the quadratic order has a dominant character and fully prevails in both $\mathrm{Col}$ and $\mathrm{K}$ phases. The fluctuations observed in the quadratic and hexatic order parameters of the system with $W_{1}=8$ (solid symbols in Fig. 8) are most probably due to a number of unresolved structural defects that persist in the $\mathrm{Col}^{-}$phase beyond our simulation time.

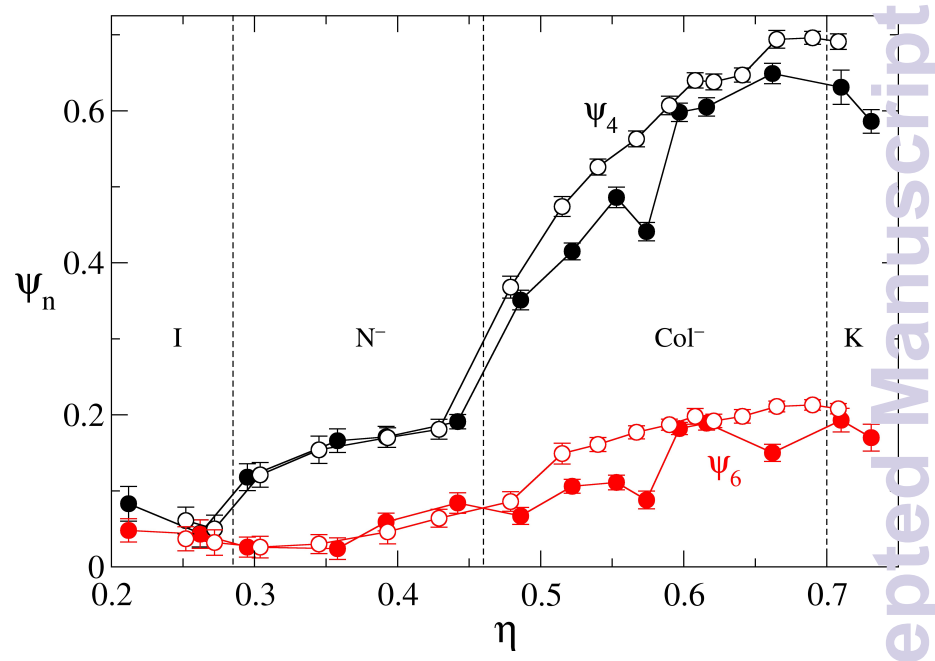

Fig. 8 Quadratic and hexatic order parameters of colloidal suspensions containing $2000 \mathrm{HBPs}$ of length $L_{1}^{*}$ and width $W_{1}^{*}=7.5$ (empty circles) and $W_{1}^{*}=8$ (solid circles) at different packing fractions.

Finally, in Fig. 9, we show the parallel (left frame) and perpendicular (right frame) radial distribution functions of a $\mathrm{K}$ phase containing short HBPs with width $W_{1}^{*}=5.5$ and packing frai tion $\eta=0.725$. The $g_{\|}(r)$ functions display well-defined periodic peaks with relatively large amplitude approximately at multipie distances of $9 T, 5.5 T$ and $T$ along the directors $\hat{\mathbf{n}}, \hat{\mathbf{m}}$ and $\hat{\mathbf{i}}$, respectively. The $g_{\perp}(r)$ functions, less intuitive than the $g_{\|}(r)$, art? the result of the combined periodicity of two peaks existing in th planes perpendicular to each director.

\subsection{Phase diagrams}

As predicted by Onsager theory ${ }^{18}$, compressing a suspension of hard-body particles promotes the transition to phases with a higher degree of orientational ordering, from isotropic to LC to crystal phases. These phase transformations are driven by an increase in excluded-volume entropy, which is sufficiently large to compensate the reduction in orientational entropy. In line with these arguments, colloidal suspensions of HBPs undergo entropydriven phase transformations that substantially depend on their geometry and, as such, can produce oblate, prolate, and biaxial 

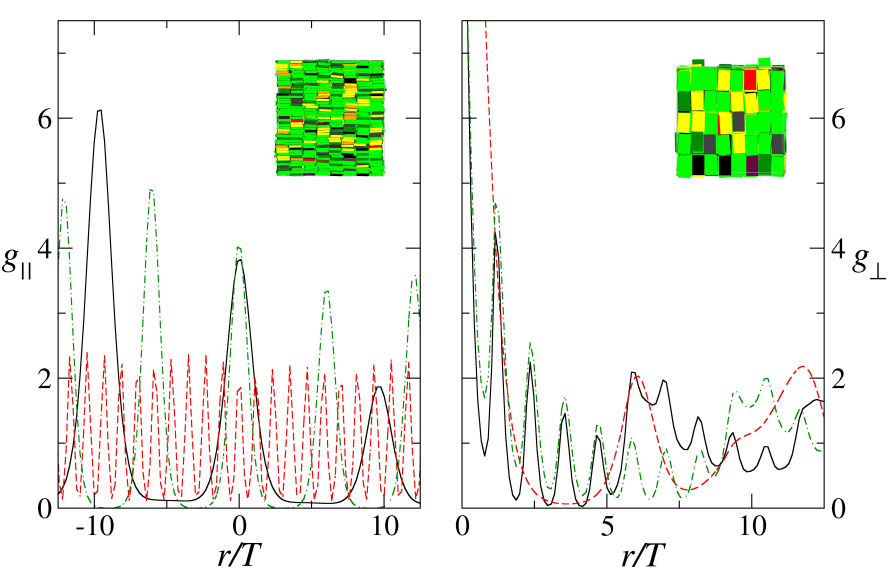

Fig. 9 Parallel (left frame) and perpendicular (right frame) pair distribution functions of a crystal phase containing 1800 HBPs of length $L_{1}^{*}$ and width $W_{1}^{*}=5.5$ at $\eta=0.725$. Solid, dot-dashed and dashed lines refer to the directions parallel (left frame) or perpendicular (right frame) to the nematic directors $\hat{\mathbf{n}}, \hat{\mathbf{m}}$ and $\mathbf{l}$, respectively. The insets show the front and top views of an equilibrium configuration, with different colours indicating the orientation of the particle major axis.

morphologies. The phase diagrams calculated by MC simulations are presented in Figs. 10 and 11 for HBPs of length $L_{1}^{*}$ and $L_{2}^{*}$, respectively. In addition, in the top frames of Figs. 12 and 13, we also show the phase diagrams for the same particle geometries as calculated from theory. The bottom frames of these figures compare the predicted boundaries of the I-N phase transition when the HBPs are assumed to be uniaxial (empty symbols) or biaxial (solid symbols).

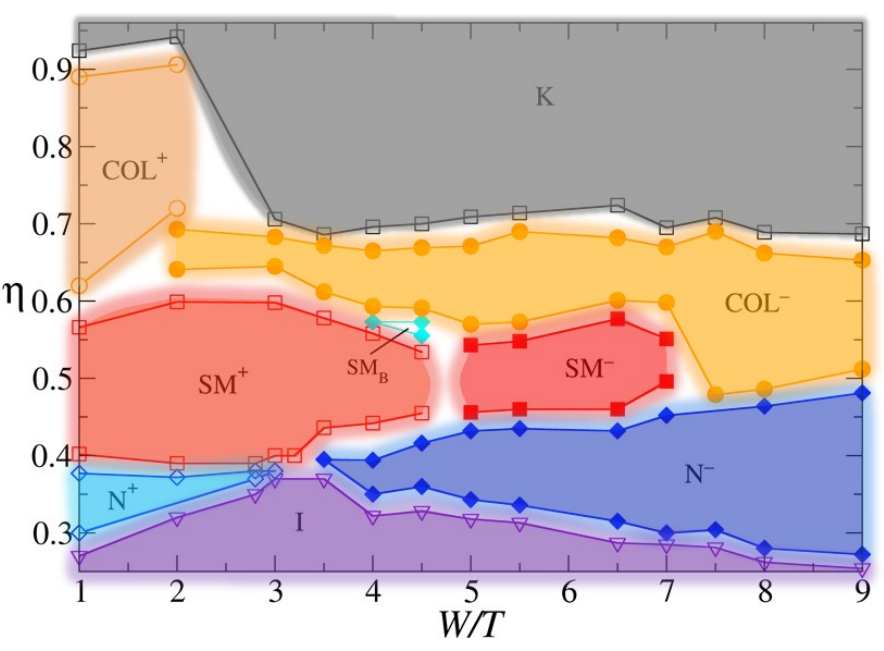

Fig. 10 Phase diagram of HBPs with length $L_{1}^{*}$, and width $1 \leq W_{1}^{*} \leq 9$.

As far as short HBPs $\left(L^{*}=9\right)$ are concerned, the simulation results in Fig. 10 indicate that the I-N phase transition occurs at packing fractions that gradually increase in the prolate region from approximately 0.28 to 0.38 , and then decrease in the oblate region down to 0.27 . Above the I-N coexistence region, which is relatively small, but significant enough to suggest a strong firstorder character, we find the $\mathrm{N}^{-}$and $\mathrm{N}^{+}$phases, respectively at $W_{1}^{*} \leq 3$ and $W_{1}^{*}>3$. The prolate $\mathrm{N}$ phase has a relatively small stability region and transforms into a $\mathrm{Sm}^{+}$phase at approximately $\eta=0.40$. The stability of the oblate $\mathrm{N}$ phase appears to increase with the particle width and at $\eta>0.40$ can transform into a $\mathrm{Sm}^{+}, \mathrm{Sm}^{-}$or $\mathrm{Col}^{-}$phase, depending on the degree of particle anisotropy. All these features are perfectly predicted by the theoretical phase diagram in Fig. 12, whose quantitative agreement with the simulation results is excellent. The only tangible discrepancy between theory and simulation arises from the location of upper phase boundary of the $\mathrm{N}$ region, which is slightly overestimated by the theory. However, we stress that in this case, we are only calculating the spinodal instability densities at which a uniaxial $\mathrm{N}$ phase consisting of aligned particles becomes unstable with respect to a layered structure. Similar considerations are still valid for the phase diagrams of long HBPs $\left(L^{*}=12\right)$, reported in Figs. 11 (simulation) and 13 (theory).

The phase diagrams of short $\left(L^{*}=9\right)$ and long $\left(L^{*}=12\right)$ HBPs exhibit a number of common features, but also some key differences mostly related to the region of stability of the nematic and columnar phases and the location of the I-N phase transition. More specifically, the I-N phase transition appears to shift to lower packing fractions at increasing particle length and, because the lower boundaries of the positionally ordered phases are not particularly influenced, the region of stability of the nematics increases too. Previous theoretical predictions based on the Zwanzig approximation locate the I-N transition of HBPs with $\left(L^{*}, W^{*}\right)=(9,3)$ at $\eta \approx 0.28^{31}$, while the results of more recent MC simulations of hard spheroplatelets, similar, but not identical, to our HBPs, are much closer to our estimations of $\eta \approx 0.35-0.40$, depending on the $L / W$ aspect ratio ${ }^{39}$. In both these works, the I-N transition was observed to be of a weak first-order, with an insignificant density difference between I and N phases. Although our theory confirms the first-order character of the I-N transition over the whole range of geometries examined, it reveals an I$\mathrm{N}$ coexistence region that is not negligible and thus suggests a rather strong first-order signature. This is also confirmed by the analysis of the equations of state (see ESI $\dagger$ ), which unveil the existence of slight discontinuities or sudden changes in the slope of $\eta v s P^{*}$. The strong first-order character of the I-N transition can dramatically determine the kinetics of formation of the $\mathrm{N}$ phase, most probably characterised by the occurrence of temporary metastable domains persisting over relatively long time scales. Further investigation is however necessary to address this qualitative intuition. The extension of the I-N coexistence region can be more easily appreciated in the bottom frame of Figs. 12 and 13, where we compare the effect of assuming a uniaxial or 
biaxial particle geometry on the location of the I and $\mathrm{N}$ phase boundaries. We notice that relatively strong first-order I-N transitions were also reported in the simulation work by John and Escobedo on systems of hard tetragonal parallelepipeds with square cross section and $L / W=6.5$ and $8^{34}$. We also notice that, according to both theory and simulation, the exact location of the I-N transition depends on the particle width across the complete range of prolate and oblate shapes, again in qualitative agreement with former simulation results on cuboids ${ }^{34}$.

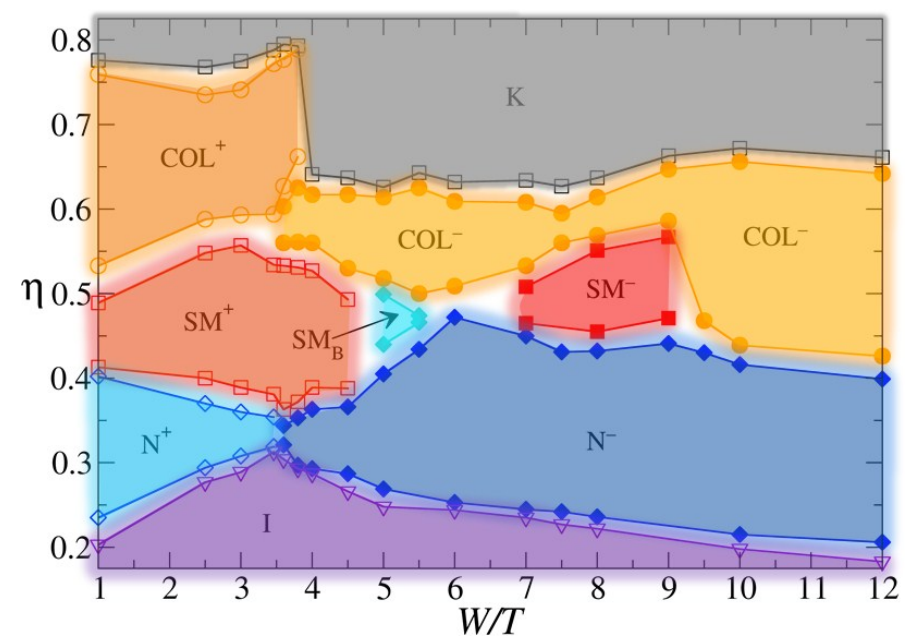

Fig. 11 Phase diagram of HBPs with thickness $T$, length $L=12 T$, and width $1 \leq W_{2}^{*} \leq 12$.

It is also interesting to highlight that assuming a uniaxial rather than biaxial geometry, when investigating the I-N transition of freely-rotating HBPs, determine a shifting of the coexistence region towards larger packing fractions. In Fig. 12b, this assumption especially influences the I-N phase boundaries of rod-like particles with square cross section, but does not have any significant effect at or close to the self-dual shape. Restricting the orientations of HBPs to six seems to have a much more relevant impact on the description of their phase behaviour than assuming them to be uniaxial.

Furthermore, our simulation and theoretical results do not provide any sound evidence of the existence of an $\mathrm{I}-\mathrm{N}_{\mathrm{B}}$ transition. The boundary between the $\mathrm{N}^{-}$and $\mathrm{N}^{+}$phases is basically located at the particle width $W=\sqrt{L T}$, corresponding to the self-dual shape at which, according to theory ${ }^{26,31,55}$ and simulation ${ }^{32,39}$ on a spectrum of hard-core biaxial particles, including HBPs, a second-order I- $\mathrm{N}_{\mathrm{B}}$ phase transition should be observed. Although our extensive simulations could not confirm the existence of the $\mathrm{N}_{\mathrm{B}}$ phase, at least for the particular board-like geometries investigated here, they revealed the formation of a $\mathrm{Sm}_{\mathrm{B}}$ phase, being approximately constrained between the $\mathrm{Sm}^{-}$and $\mathrm{Sm}^{+}$phases and consisting of HBPs with $W \approx 1.4 \sqrt{L T}$. The eventual occurrence of

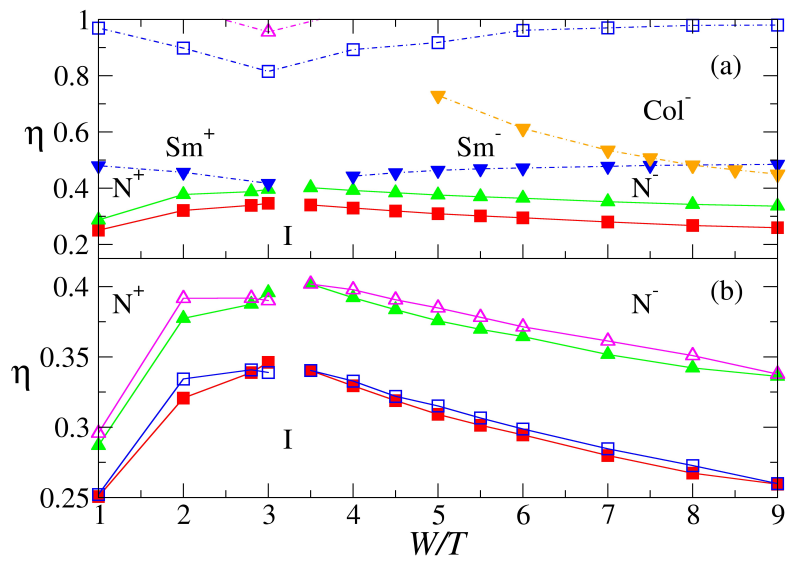

Fig. 12 (a) Phase diagrams for HBPs with $L_{1}^{*}$ calculated from theory. Red squares and green triangles denote the I-N phase transition, blue and orange inverted triangles denote, respectively, the N-Sm and N-Col instability densities, and blue open squares and pink open triangles denote the $\mathrm{N}_{U}-\mathrm{N}_{\mathrm{B}}$ transition. (b) Comparison of the predicted I-N phase transition found when considering the board particles as biaxial (solid symbols) and as uniaxial (empty symbols). For the uniaxial predictions, we ignore ordering of the angle $\gamma$ (rotations about the particle long-axis).

the $\mathrm{N}^{-}-\mathrm{N}_{\mathrm{B}}$ and $\mathrm{N}^{+}-\mathrm{N}_{\mathrm{B}}$ phase transitions were examined theoret ically, by keeping one of the particle axes perfectly aligned with the nematic director and allowing the other two particle axes tn reorient. In particular, we observed that the $\mathrm{N}_{\mathrm{B}}$ is pre-empted by the Sm phase over all its range and cannot form unless the $\mathrm{Sm}$ phase is somehow destabilised (see Figs. 12a and 13a). W. did not examine the range of stability of the Sm phase, but oniv the spinodal instability boundaries at which a perfectly aligner. $\mathrm{N}_{\mathrm{U}}$ phase first becomes unstable with respect to a layered stru ture. Our predictions locate the $\mathrm{N}$-Sm instability of short $\mathrm{HB}$. at $0.42<\eta<0.48$, depending on the particle width (blue in verted triangles in Fig. 12a), in very good agreement with the $\mathrm{N}^{+}-\mathrm{Sm}^{+}$and $\mathrm{N}^{-}-\mathrm{Sm}^{-}$phase boundaries reported in Fig. 10. As far as long HBPs are concerned, our theory predicts the occl. rence of these density instabilities at similar packing fractions, that is $0.42<\eta<0.49$, depending on $W_{2}^{*}$ (blue inverted triangles in Fig. 13a), again in excellent agreement with the simulation results. It should be noticed that the N-Sm instability densities at $W_{1}^{*}=3.5$ and $4 \leq W_{2}^{*} \leq 5$ are missing in Figs. 12a and 13a, respectively. Our theory cannot account for the $\mathrm{N}^{-}-\mathrm{Sm}^{+}$(oblateto-prolate) phase transition, which is observed in simulations, but would only provide the instability density of a hypothetical transition to an oblate smectic phase. Aware of the limitations of our theory, we have therefore decided to omit these points.

At sufficiently large packing fractions, both oblate and prolate 


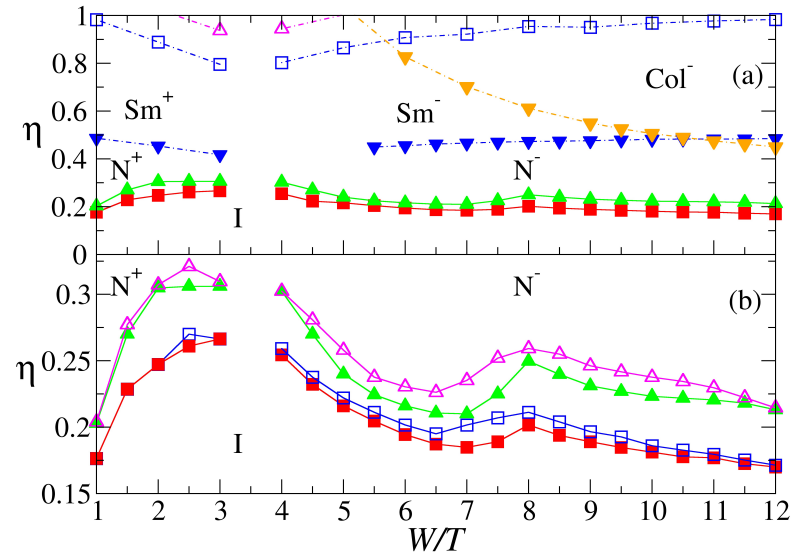

Fig. 13 (a) Phase diagrams for HBPs with $L_{2}^{*}$ calculated from theory. Red squares and green triangles denote the I- $\mathrm{N}$ phase transition, blue and orange inverted triangles denote, respectively, the N-Sm and N-Col instability densities, and blue open squares and pink open triangles denote the $\mathrm{N}_{U}-\mathrm{N}_{B}$ transition. (b) Comparison of the predicted I-N phase transition found when considering the board particles as biaxial (solid symbols) and as uniaxial (empty symbols). For the uniaxial predictions, we ignore ordering of the angle $\gamma$ (rotations about the particle long-axis).

HBPs can form Col phases. The region of existence of the Col phase and its stability with respect to the Sm phases is very similar for short and long HBPs. The most remarkable difference to mention is the ability of short HBPs to self-assemble into $\mathrm{Col}^{-}$phases in a region of the phase diagram where long HBPs can only form $\mathrm{Col}^{+}$phases, that is for $W<\sqrt{L T}$. More generally, the intriguing result of oblate (prolate) HBPs to self-assemble into prolate (oblate) phases is also detected at lower packing fractions, where oblate HBPs $(W>\sqrt{L T})$ can aggregate into $\mathrm{Sm}^{+}$phases. As a consequence of this peculiar behaviour, some particle geometries undergo oblate-prolate-oblate transitions at increasing pressure. This is for instance the case of systems with particle of size $\left(L_{1}^{*}\right.$, $\left.W_{1}^{*}\right)=(9,4)$ and $\left(L_{2}^{*}, W_{2}^{*}\right)=(12,4.5)$, where an $\mathrm{N}^{-}-\mathrm{Sm}^{+}$followed by a $\mathrm{Sm}^{+}-\mathrm{Col}^{-}$phase transition is observed. Even more intriguing is the sequence of phase transitions observed for HBPs of size $\left(L_{2}^{*}\right.$, $\left.W_{2}^{*}\right)=(12,3.8)$, which undergo a remarkable $\mathrm{N}^{-}-\mathrm{Sm}^{+}-\mathrm{Col}^{-}-\mathrm{Col}^{+}$ transformation across the range $0.35<\eta<0.66$.

The relative Col/Sm phase stability in suspensions of polydisperse colloidal platelets has recently been investigated by density functional theory within the FMT formalism ${ }^{56}$. In monodisperse systems, the particle geometry play a dominant role across the range of anisotropies studied here. Both short and long HBPs are able to self-assemble into Col phases regardless of their prolate or oblate geometry. In addition, for particularly pronounced oblate anisotropies, at which $L / W<1.3$, the range of stability of the $\mathrm{Col}^{-}$phase increases significantly and a direct $\mathrm{N}^{-}-\mathrm{Col}^{-}$ transformation, without the occurrence of the $\mathrm{Sm}^{-}$phase, is observed. Our theoretical predictions in Figs. 12a and 13a show that the $\mathrm{N}^{-}-\mathrm{Col}^{-}$transition is indeed favoured for plate-like particles, whose nematic-columnar instability density is found to be lower than the nematic-smectic instability density at $W_{1}^{*}>7.5$ and $W_{2}^{*}>10$. Depending on the particle width, the columnar phase can be obtained by compressing $\mathrm{N}^{-}, \mathrm{Sm}^{-}, \mathrm{Sm}_{\mathrm{B}}$ or $\mathrm{Sm}^{+}$phases. The occurrence of all these phase transitions is determined over all the particle width analysed here, from prolate $(W<\sqrt{L T})$ to oblate $(W>\sqrt{L T})$ geometries. HBPs with a quasi self-dual shape, for instance $\left(L_{2}^{*}, W_{2}^{*}\right)=(12,3.6)$, are very peculiar because at increasing packing, they can orient along either their shortest or longest axis or both and thus can switch from prolate to oblate to biaxial phases. In the range $\sqrt{L T}<W<4.5 T$, these particles undergo a $\mathrm{N}^{-}$to $\mathrm{Sm}^{+}$transition first and then, at larger pressures, a transition from the $\mathrm{Sm}^{+}$to the $\mathrm{Col}^{-}$phase, which has a significant degree of biaxiality. Since we did not study the details of the kinetics of $\mathrm{Sm}^{+}$to $\mathrm{Col}^{-}$phase transformation, we can only speculate that an in-layer reorganisation of the particles takes place at increasing densities and promotes a gradual micro-segregation of the smectic layers in separate stacks. Also prolate HBPs are able to form columnar phases, as was previosly observed in systems of hard tetragonal parallelepipeds ${ }^{34}$. The $\mathrm{Col}^{+}$phase is stable at relatively high density, especially in systems of short HBPs, where the transition to the $\mathrm{K}$ phase is only found at $\eta>0.9$. By contrast, to the best of our knowledge, uniaxial particles are not able to form Col phases ${ }^{38,57}$, unless a degree of size dispersity ${ }^{58,59}$ or flexibility ${ }^{60}$ is incorporated.

\section{Conclusions}

In summary, we have investigated the phase behaviour and mapped out the phase diagram of colloidal suspensions of short and long HBPs, which, depending on their length-to-width ratio, are able to self-assemble into oblate, prolate, and biaxial phases with a large degree of positional and/or orientational order. We have characterised these phases by calculating uniaxial and biaxial order parameters as well as the structural correlation between the particles along the phase directors and perpendicularly to them. Short and long HBPs exhibit similar phase diagrams, with an I-N phase transformation at approximately $0.2<\eta<0.3$, depending on the particle width, and a transition from prolate to oblate nematic LCs at the so-called self-dual shape, where $W=\sqrt{L T}$. Our theory, a generalisation of Onsager theory to incorporate particle biaxiality, is able to capture both these features very well. In addition, it reveals an I-N coexistence region that is significantly larger than that predicted by previous studies on identical $^{29,31}$ or similar ${ }^{26,39}$ particle geometries, but in agreement with the simulations by John and Escobedo on hard tetragonal parallelepipeds ${ }^{34}$. This coexistence region, which reveals a 
relatively strong first-order character, is virtually independent on the particle width across the complete range of prolate and oblate shapes investigated here.

More surprisingly, we did not observe, neither by simulations nor theory, the formation of a stable biaxial nematic phase. While at the self-dual shape and sufficiently large densities a secondorder I- $\mathrm{N}_{\mathrm{B}}$ transition is generally predicted by theory ${ }^{26,31,55}$, our HBPs undergo transitions from I to $\mathrm{N}_{\mathrm{U}}$ to Sm phase and no evidence of biaxiality is observed. We notice that, by limiting the particle orientations to six, these theories locate the I-N transition at packing fractions $\eta<0.3$ at which we still observe I phases. At such moderate densities, the formation of a Sm phase, which we only detect at $\eta \geq 0.4$, is very unlikely and consequently the $\mathrm{N}_{\mathrm{B}}$ phase would be indirectly favoured. We conclude that a biaxial geometry, per se, might not be enough to observe biaxial phases and a degree of size dispersity, as reported experimentally ${ }^{28}$ and suggested by theory 27,29 , is crucial to weaken the stability of the Sm phase and thus create the best conditions to form the $\mathrm{N}_{\mathrm{B}}$ phase. Contrary to the $\mathrm{N}_{\mathrm{B}}$, the $\mathrm{Sm}_{\mathrm{B}}$ phase has been detected for the two particle lengths $L_{1}$ and $L_{2}$, although within a very small range of stability.

Finally, we have also shed light on the existence of the discotic (or oblate) smectic phase. The $\mathrm{Sm}^{-}$phase, which consists of particles with their long axes randomly oriented within the smectic layers, has been found in the phase diagram of both short and long HBPs. Although the $\mathrm{Sm}^{-}$phase had been predicted by the FMT and lattice MC simulations, in both cases within the Zwanzig approximation ${ }^{36,37}$, it was not observed in off-lattice MC simulations of freely rotating hard parallelepipeds with square cross sec$\operatorname{tion}^{34,35}$. The latter geometry promotes instead the formation of a cubatic-like mesophase, the so-called parquet phase, where the particles are arranged in stacks oriented roughly perpendicular to one another and without formation of layers ${ }^{34,35}$. The board-like geometry of our particles, very different from that of a cuboid, does not promote the formation of this phase, but can definitively confirm the existence of the $\mathrm{Sm}^{-}$phase.

\section{Acknowledgments}

AC acknowledges project CTQ2012-32345 funded by the Junta de Andalucía-FEDER and C3UPO for HPC facilities provided. MD gratefully acknowledges funding from the Alexander von Humboldt Foundation. AP acknowledges financial support from EPSRC under grant agreement EP/N02690X/1 and a Researcher Mobility Fellowship awarded by the Royal Society of Chemistry for funding his research stay at the Department of Physical, Chemical and Natural Systems, Pablo de Olavide University. AP and AM would like to acknowledge the assistance given by IT Services and the use of the Computational Shared Facility at the University of Manchester.

\section{References}

1 R. G. Jones, E. S. Wilks, W. V. Metanomski, J. Kahovec, M. Hess, R. Stepto, T. Kitayama, eds. Compendium of Polymer Terminology and Nomenclature: IUPAC Recommendations 2008, RSC Publishing, 2009, 11, 213. ISBN 978-0-85404-491-7.

2 R. Brown, in The Miscellaneous Botanical Works of Robert Brown, edited by John J. Bennett, Vol. 1 (London, 1866).

3 J. G. C. Veinot, H. Yan, S. M. Smith, J. Cui. Q. Huang and T. J. Marks, Nano Letters, 2002, 2, 333-335.

4 A. van Blaaderen, Nature, 2006, 439, 545-546.

5 P. A. Buining, C. Pathmamanoharan, J. B. H. Jansen, and H. N. W. Lekkerkerker, JACS, 1991, 74, 1303-1307.

6 M. Adams, Z. Dogic, S. L. Keller, and S. Fraden, Nature, 1998 393, 349-352.

7 F. M. van der Kooij, K. Kassapidou, and H. N. W. Lekkerkerker Nature, 2000, 406, 868-871.

8 Y. Sun and Y. Xia, Science, 2002, 298, 2176-2179.

9 V. N. Manoharan, M. T. Elsesser, and D. J. Pine, Science, 301 2003, 483-487.

10 S. S. Shankar, A. Rai, B. Ankamwar, A. Singh, A. Ahmad, and M. Sastry, Nat. Mat., 2004, 3, 482-488.

11 L. Rossi, V. Soni, D. J. Ashton, D. J. Pine, A. P. Philipse, P. M Chaikin, M. Dijkstra, S. Sacanna, and W. T. M. Irvine, PNAS 2015, 112, 5286.

12 S. Sacanna, W. T. M. Irvine, P. M. Chaikin, and D. J. Pine Nature, 2010, 464, 575.

13 S. Sacanna, M.Korpics, K. Rodriguez, L. Colón-Meléndez, S. H. Kim, D. J. Pine, and G.-R. Yi, Nat. Commun., 2013, 4, 1688.

14 Y. Xiang, X. Wu, D. Liu, X. Jiang, W. Chu, Z. Li, Y. Ma, W Zhou, and S. Xie, Nano Lett., 2006, 6, 2290-2294.

15 Y. Okuno, K. Nishioka, A. Kiya, N. Nakashima, A. Ishibashia and Y. Niidome, Nanoscale, 2010, 2, 1489-1493.

16 M. B. Cortie, F. Liu, M. D. Arnold, and Y. Niidome, Langmu.. 2012, 28, 9103-9112.

17 B. N. Khlebtsov, Z. Liuc, J. Yec, N. G. Khlebtsov, J. Quan . Spectrosc. Radiat. Transf., 2015, 167, 64-75.

18 L. Onsager, Ann. N.Y. Acad. Sci., 1949, 51, 627-659.

19 B. Ruzicka, E. Zaccarelli, L. Zulian, R. Angelini, M. Sztucki, A. Moussaïd, T. Narayanan, and F. Sciortino, Nat. Mater., 2011, 10, 56-60.

20 D. J. Ashton, R. L. Jack, and N. B. Wilding, Phys. Rev. Lett., 2015, 114, 237801.

21 S. C. Glotzer and M. J. Solomon, Nat. Mat., 2007, 6, 557-562.

22 P. F. Damasceno, M. Engel, and S. C. Glotzer, ACS Nano, 2012, 6, 609-614.

23 G. van Anders, N. K. Ahmed, R. Smith, M. Engel, and S. C. Glotzer, ACS Nano, 2014, 8, 931-940.

24 B. de Nijs, S. Dussi, F. Smallenburg, J. D. Meeldijk, D. J. Groe- 
nendijk, L. Filion, A. Imhof, A. van Blaaderen, and M. Dijkstra, Nat. Mat., 2015, 14, 56-60.

25 C. Avendaño, G. Jackson, E. A. Müller, and F. A. Escobedo, PNAS, 2016, 113, 9699-9703.

26 M. P. Taylor and J. Herzfeld, Phys. Rev. A, 1991, 44, 37423751.

27 A. G. Vanakaras, M. A. Bates, and D. J. Photinos, Phys. Chem. Chem. Phys., 2003, 5, 3700-3706.

28 E. van den Pol, A. V. Petukhov, D. M. E. Thies-Weesie, D. V. Byelov, and G. J. Vroege, Phys. Rev. Lett., 2009, 103, 258301.

29 S. Belli, A. Patti, M. Dijkstra, and R. van Roij, Phys. Rev. Lett., 2011, 107, 148303.

30 R. Zwanzig, J. Chem. Phys., 1963, 39, 1714-1721.

31 S. Belli, M. Dijkstra, and R. van Roij, J. Phys.: Condens. Matter, 2012, 24, 284128.

32 P. Camp and M. Allen, J. Chem. Phys., 1997, 106, 6681-6688.

33 S. D. Peroukidis and A. G. Vanakaras, Soft Matter, 2013, 9, 7419-7423.

34 B. S. John and F. A. Escobedo, J. Phys. Chem. B, 2005, 109, 23008-23015.

35 B. S. John, C. Juhlin, and F. A. Escobedo, J. Chem. Phys., 2008, 128, 044909.

36 A. Casey and P. Harrowell, J. Chem. Phys., 1995, 103, 6143.

37 Y. Martínez-Ratón, Phys. Rev. E, 2004, 69, 061712.

38 L. Mederos, E. Velasco, and Y. Martínez-Ratón, J. Phys.: Condens. Matter, 2014, 26, 463101.

39 S. D. Peroukidis, A. G. Vanakaras, and D. J. Photinos, Phys. Rev. E, 2013, 88, 062508.

40 P. A. C. O'Brien, M. P. Allen, D. L. Cheung, M. Dennison and A. Masters, Soft Matter, 2011, 7, 153-162 .

41 S. Gottschalk, M. C. Lin, and D. Manocha, Comput. Graph., 1996, 30, 171-180.
42 R. Eppenga and D. Frenkel, Mol. Phys., 1984, 52, 1303-1334.

43 P. I. C. Teixeira, M. A. Osipov, and G. R. Luckhurst, Phys. Rev. E, 2006, 73, 061708.

44 M. P. Allen, Liq. Cryst., 1990, 8, 499.

45 P. Camp, M. P. Allen, and A. Masters, J. Chem. Phys., 1999, 111, 9871.

46 F. H. Ree and W. G. Hoover, J. Chem. Phys., 1964, 40, 939.

47 F. H. Ree and W. G. Hoover, J. Chem. Phys., 1967, 46, 4184.

48 A. Y. Vlasov, X. M. You, and A. J. Masters, Mol. Phys., 2002, 100, 3313.

49 B. Mulder, Phys. Rev. A, 1987, 35, 3095.

50 R. van Roij, P. Bolhuis, B. Mulder, and D. Frenkel, Phys. Rev. E, 1995, 52, R1277.

51 J. S. van Duijneveldt and M. P. Allen, Mol. Phys., 1997, 90, 243.

52 A. Patti, D. El Masri, R. van Roij, and M Dijkstra, J. Chem. Phys., 2010, 132, 224907.

53 A. Donev, J. Burton, F. H. Stillinger, and S. Torquato, Phys. Rev. B, 2006, 73, 054109

54 K. W. Wojciechowski and D. Frenkel, Comput. Methods Sci. Technol., 2004, 10, 235.

55 Y. Martínez-Ratón, S. Varga and E. Velasco, Phys. Chem. Chem. Phys., 2011, 13, 13247-13254.

56 E. Velasco and Y. Martínez-Ratón, Phys. Chem. Chem. Phys., 2014, 16, 765.

57 P. Bolhuis and D. Frenkel, J. Chem. Phys., 1997, 106, 666.

58 A. Stroobants, Phys. Rev. Lett., 1992, 69, 2388.

59 E. Paineau, M.-E. M. Krapf, M.-S. Amara, N. V. Matskova, I. Dozov, Stéphan Rouzière, A. Thill, P. Launois and P. Davidson, Nat. Comm., 2016, 7, 10271.

60 E. Grelet, Phys. Rev. Lett., 2008, 100, 168301. 

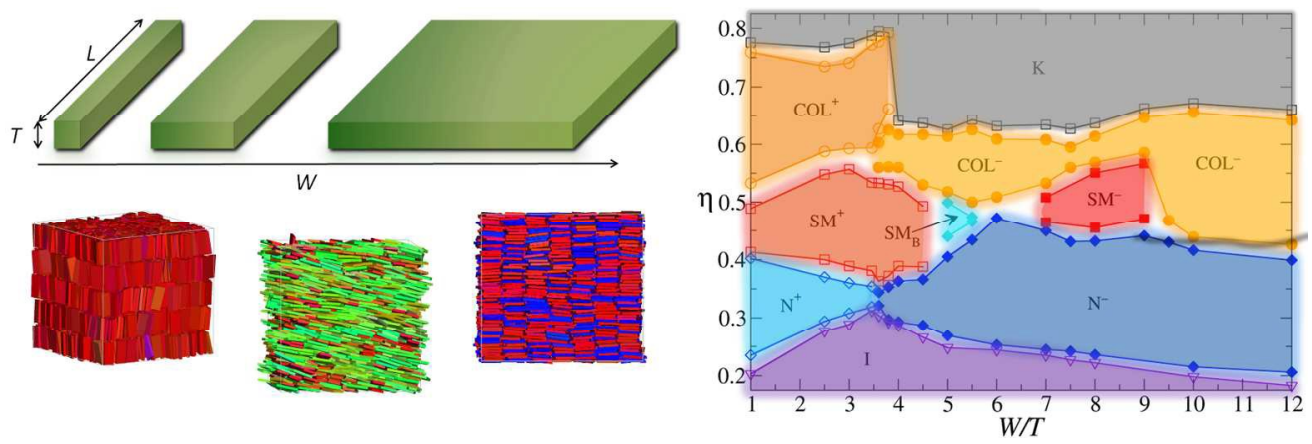